\title{
APPLICATIONS OF SRD-METHOD IN HORTICULTURAL AND FOOD
} SCIENCES

\author{
Ildikó FruZsina Boros ${ }^{1,2}$, NoÉmi KAPPel ${ }^{2}$, Krisztina MAdaras ${ }^{3}$, AtTila Gere $^{1}$, \\ LÁSZLÓ SIPOS ${ }^{1}$ \\ ${ }^{1}$ Department of Postharvest and Sensory Evaluation, Faculty of Food Science, Szent \\ István University, 29-43 Villányi Street, H-1118 Budapest, Hungary \\ ${ }^{2}$ Department of Vegetable and Mushroom Growing, Faculty of Horticultural Science, \\ Szent István University, 29-43 Villányi Street, H-1118 Budapest, Hungary \\ ${ }^{3}$ Department of Ecological and Sustainable Production Systems, Szent István University, \\ 29-43 Villányi Street, H-1118 Budapest, Hungary \\ boros.ildiko.fruzsina@kertk.szie.hu
}

\begin{abstract}
Scientific questions in horticultural and food sciences can be classified by their complexity. On the one hand, the effect of an attribute is evaluated without changing other factors. The other type is when the effect of the interactions of different treatments is analysed. In some cases, it would be necessary to use new approaches. How can we evaluate cultivars, methods, proceedings, treatments, etc. meanwhile using all parameters at the same time? Sum of Ranking Differences (SRD) is an alternative statistical method, implemented by Héberger (2010). Validation and the software implementation was done by HÉBERGER AND KOLLÁR-HUNEK (2011). Cultivars, methods, procedures, treatments, etc. can be compared successfully with SRD-method. Several international publications proved the relevancy of the methodology. In this study, SRD-method is introduced, as well as those researches, which conducted in horticultural and food sciences. Based on these, new fields of application are suggested.
\end{abstract}

Keywords: sum of ranking differences (SRD), rank order, nonparametric tests, multicriteria optimization

\section{INTRODUCTION}

In the horticultural and food sciences, the subject of the research could be analysed from one or more aspects in accordance to the experimental design. These analyses are primarily used for the comparison of substances in question, which solutions are based on parametric statistical methods and post-hoc tests, which can only be used after the criteria tests.

The most commonly used statistical tests (t-test, analysis of variance (ANOVA)) require normally distributed variables. In the case of normal distribution, the mean, the median and the mode are in the same place, ergo they are equal (Gaussian curve). In many cases, skewness and kurtosis could be present, therefore the average, the median and the mode relation can be deceiving. If the value of kurtosis and/or skewness exceeds \pm 1 , then the distribution cannot be considered as normal. For values of skewness and kurtosis, and the quotient of their standard error do not allowed to exceed \pm 1.96 (TABACHNICK AND FIDELL, 2003, 2007). In addition to the graphical test methods, the most commonly used tests are Kolmogorov-Szmirnov and Shapiro-Wilk tests to test the normal distribution. For normality testing, the null hypothesis is that the distribution of the examined variable does not differ from the normal distribution. If the conditions of normality are not fulfilled we can choose from several possible solutions: 1. transforming the data, which converts them into normal distribution (square root, logarithmic, 1/x, Box-cox etc. transformations), 2. use of nonparametric tests, which are equivalent to the parametric ones (e.g. Two-sample $t$ test $\rightarrow$ Mann-Whitney U-test, one-way analysis of variance $\rightarrow$ Kruskal-Wallis test). 
It is generally accepted that nonparametric tests have less statistical power than parametric ones. In order to be able to make a decision about the conditions of normality, at least 10 parallel measurements are required. In the practice of horticultural and food sciences this requirement is usually not fulfilled. In case of low number of cases, the normality requirements can be harmed. As a result, the requirements of parametric tests are not fulfilled, so the less efficient, but the distribution independent nonparametric methods should be chosen, which are not sensitive to damage the normality conditions and distribution of the samples variety (ANDRIĆ AND HÉBERGER, 2015).

In the horticultural and food sciences, typically one factor effect is tested and all other factors remain unchanged. The advantage of this practice is that we can clearly identify that factor which is responsible for the change, but we do not have any knowledge about what outcome would be gained whether more or all factors would be taken into account. Therefore, a new approach is needed that takes into account all the measured features together, creating more reliable results. Accordingly, due to the new approach, multivariate nonparametric statistical methods come into prominence.

\section{MATERIAL AND METHOD}

The principle of 'Sum of Ranking Differences, SRD' was created by HÉBERGER (2010), its validation and software implementation was realized by HÉBERGER AND KOLLÁR-HUNEK (2011), which enabled the separation of different methods and procedures based on the principle of the method. The presentation of the methodology is based on their research results. Objects (statistical cases, compounds/components) are sorted in rows, the variables (models, methods) are arranged in the input matrix columns, will be aligned in increasing order based on the results of the objects. Then the absolute values of different standards (reference) and individual ranking differences will be calculated and summarized for each variable/model. Different standard (reference) values can be: average (AVE), maximum (Max), minimum (Min), or scanned value (Read). Thus, the difference between the rankings, the SRD values are calculated for each variable/model (Figure 1).

Calculations are be performed in Microsoft Office Excel 2007 macro, which is written in Visual Basic programming language (freely available here): http://aki.ttk.mta.hu/srd/. The method is based on that the value is even more similar to the evaluated attribute how closer is to the reference/standard SRD-value. The SRD-method validation was conducted with permutation tests by using 3 million simulated compare ranks with random numbers (CRRN).

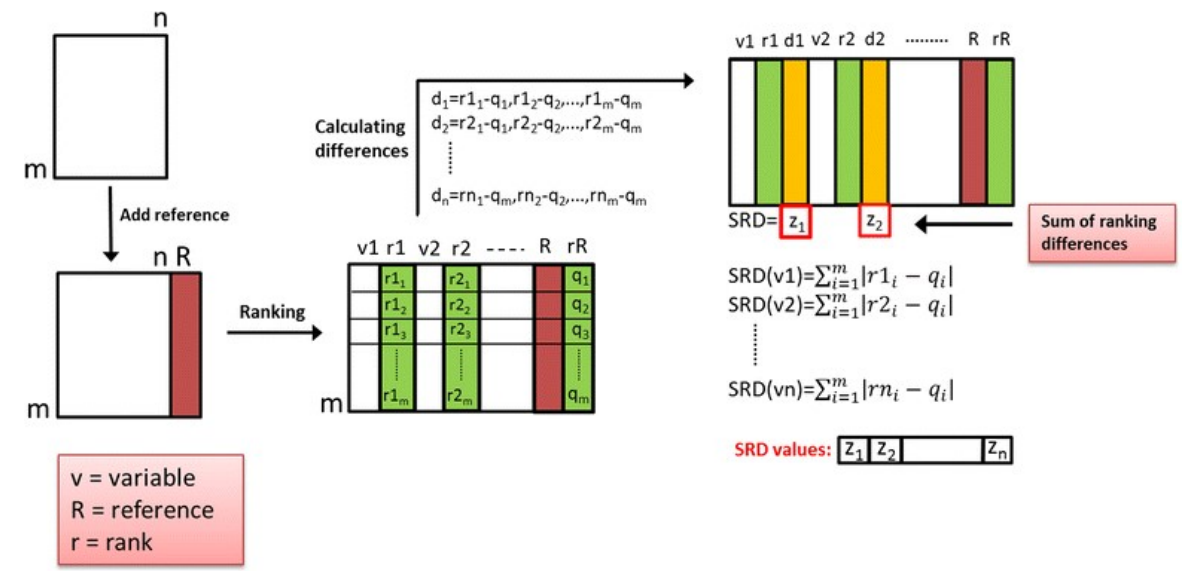

Figure 3. Calculation of SRD values

Source: BAJUSZ ET AL. (2015) 
In the case of a low number of cases a recursive algorithm based on the theoretical distribution is calculated, with which the distribution function can be converged. In the case of a higher case number $(n>8)$, the normal distribution, as a good approximation, can be used for the description of the theoretical (random) SRD distribution function. The normal approximation of the theoretical distribution of SRD values was introduced by HÉBERGER and KOLLÁR-HUNEK (2011), as well as the improved version, which handles ties present in a dataset. With the increase of $n$, the approximation of normal distribution gives better results (KOLLÁR-HUNEK and HÉBERGER, 2013). The results are rank order (SRD values). When visualizing the results, the normal approximation values are read on the right abscissa, while the SRD\% values are shown on the left abscissa and on the ordinate. These SRD values are usually normalized to enable the comparison of different SRD calculations.

Leave-one-out (LOO) cross-validation process is a possibility for the variability of the SRD values, where one row is always removed from the dataset and a separate SRD is run on each of them. In the nonparametric way, Wilcoxon matched pairs test is a process for the pairwise comparison of the SRD values generated from the cross-validation process.

\section{RESULTS}

\section{Applications}

Several publications show that Sum of Ranking Difference has justification in horticultural and food sciences. Applications of SRD method with or without the combination of analysis of variance were successfully used for the assessment of methods and models (selection of chromatographic columns) (HÉBERGER, 2010), for the investigation of sensory panel performance (SIPOS ET AL., 2011), for the comparison of multiclass classifiers (SZÖLLŐSI ET AL., 2012), for industrial preference mapping (LOSÓ ET AL., 2012), for the comparison of chemometric methods (FRAGKAKI ET AL., 2012), for the evaluation of the results of collaborative laboratory trials (ŠKRBIĆ ET AL., 2013), for evaluation of single-cell gel electrophoresis (HÉBERGER ET AL., 2014), for chromatographic analysis of lipophilicity (ANDRIĆ AND HÉBERGER, 2015) for to choose the best models to predict consumer choices during eye-tracking studies (GERE ET AL., 2016).

Newest publications showed that SRD could be a good option for research analysis from one or more aspects.

CSAMBALIK ET AL. (2017) identified a combination of methodologies, that could be validated to be able to do multidimensional evaluation for the investigation necessary of phytonutrient content in tomato landraces. Based on the phytonutrient (FRAP, DPPH, sugar-acid ratio, dry matter content, vitamin $\mathrm{C}$ content, total phenolic content, lycopene content) content of landraces (rows) and commercial tomato varieties (columns) they made a ranking with SRD-method, where the maximum phytonutrient values were used for reference column. They also used leave-one-out (LOO) cross-validation process, where one row (phytonutrient) was always removed from the dataset and after that Wilcoxon matched pairs test was run. The results of the Wilcoxon matched pairs test showed that landraces have the most favourable phytonutrient value. According to their results, three groups have been identified; Bugac and Veresegyháza were the closest to the reference landrace that had the highest phytonutrient values. These were followed by Máriapócs, Kozárd, Soltvadkert, Gyöngyös, Cigánd, Jánoshalma landraces, and the Hellfrucht variety. Nagykáta and Cegléd landraces were the last group. Bugac did not shows significant difference from Veresegyház $(p<0.05)$. Nagykáta and Cegléd landraces were not significantly different from Máriapócs and Hellfrucht. 
SIPOS ET AL. (2017) assessed 11 cherry tomatoes from commercial trade. Lycopene content was measured as well as the FRAP-, DPPH-, CUPRAC-, ABTS- and TPC-assay following extraction by either water, ethanol or methanol. SRD was used to rank the antioxidantcapacity assay and extraction eluent combinations. Different assays are capable of the most efficient differentiation between the samples by the application of the different extraction eluents during the sample preparation. As reference, the theoretical best method extraction combination was defined (in this case the ideal maximum value represents the idealistic antioxidant capacity assay extraction combination). The CUPRAC-EtOH and the CUPRAC-supernatant assay combinations were the most effective among the tested methods. This method is a relatively rarely applied compared to DPPH or FRAP, which proved to be far less capable of distinguishing cherry tomato samples.

GERE ET AL. (2017) worked on an approach, that uses a bunch of multivariate statistical methods to identify the key JAR variables for product development. Evaluation of the connection between JAR and hedonic data using multiple methods. The authors also selected the best evaluation method(s) for JAR analysis.

ASTM MNL-63 consisted of the evaluations of five products using six JAR variables along with one overall liking variable. During the ranking of attributes the maximum values of the rows were used for reference column. After the normalization and calculations, they identified that the following attributes had the most important impact on liking $(p<0.05)$ : Flavour-, Stickiness + and Color- .

Different Penalty analysis evaluation methods were used in the rows in SRD after normalization of the input data matrix (square root transformation of the original attributes). The reference column contained the maximum values of the rows during SRDmethod. Result showed GPCM and OLS were the closest to the zero point; other methods located over the five percentiles line; these had no significant evidence to their rank.

SIPOS ET AL. (2016) evaluated eight different Ocimum basilicum L. gene bank accessions with SRD-method and also applied LOO, Wilcoxon and Sign tests. SRD was run based on vitamin C, DPPH, total phenolic content, FRAP, total flavonoid, salvigenin, nevadensin, essential oil contents. For the reference column the maximum values of the attributes were used. Results showed that 'M. Grünes' was the best variety, followed by 'A-1'with 'Dark Opal'. The weakest groups were 'Arvada', 'Genovese', 'Lengyel', 'Piros' and Rit-Sat' genotypes which were no significantly different from each other.

Boros ET AL. (2016) ranked lettuce types with SRD-method. Crisphead, Butterhead, Romaine, Green and Red leaf types of lettuce were evaluated about their bioactive compounds (iron, folate, vitamin $\mathrm{C}, \beta$-carotene, lutein and total phenolic content). For the SRD-method, bioactive compounds were used in rows and columns contained lettuce types. Based on the results, Romaine and Red leaf lettuce were the best, these were followed by Butterhead and Green leaf types. The weakest was the Crisphead type.

RÁCZ ET AL. (2015) compared and evaluated (ranked and grouped) antioxidant capacity assays of berries and sour cherries. They determined which assay(s) can be used with the least error, if only one technique can be chosen. The average was chosen as reference for all of the datasets. In addition, SRD could rank the different antioxidant activity methods for berry and sour cherry samples. For both datasets FRAP and TPC were the closest to reference, while ACW and ACL were the fairest. (In the case of berries the rank was FRAP, TRSC, TPC, DPPH, ACL, ACW. In the case of sour cherry the rank was TPC, FRAP and TEAC, ACL, ACW.) FRAP and TPC were recommended to substitute all the other antioxidant capacity methods for both datasets. 


\title{
DISCUSSION
}

In this study, we showed the opportunities of SRD-method in horticulture and food sciences. At this moment, the software which capable and incapable to handle repetitions is freely available here: http://aki.ttk.mta.hu/srd/. Also, there are input and output files presenting the methodology.

Simultaneous evaluation of different attributes gives the importance of weighting because every attribute has dissimilar relevancy. Calculation with the weight is not solved neither in theoretic way nor in software programming level. It gives more difficulties that maximum 40 rows could be taken to the input matrix if necessary the permutation tests by using 3.000.000 simulated compare ranks with random numbers (CRRN), but without this validation the software is possible to handle 1500 rows for simple ranking. In that case, it could be more powerful to use different validation method. The SRD program with ties has relative long calculation time caused by the simulation of a 3 million $n$-dimensional random vector set, that is indispensable to each $n$-dimensional IRC vector (Index vectors of Reference Column), given to an input matrix, where $n>8$. KOLLÁR-HUNEK AND HÉBERGER (2015) suggested the optimization of the probability distributions for the faster run. At this moment the base value of the ranking can handle 2 or 3 decimal places. If the original data were not measured in the same scale, then it is necessary to do data transformation, meanwhile the method is sensitive for this. Usually logarithmic or root transformation give good results.

In point of fact Sum of Ranking Differences (SRD) method is useful for every scientific question where it is necessary the ranking and simultaneously available several attributes (with equal weight). Expedience to use both validation method ( 3 million size $n$ dimensional random vector set curve, leave-one-out (LOO) cross-validation process and nonparametric Wilcoxon matched pairs test).

\section{ACKNOWLEDGEMENTS}

\author{
F) \\ Supported by the ÚNKP-16-4 and ÚNKP-17-4 New National Excellence Program \\ of the Ministry of Human Capacities.
}

\section{REFERENCES}

ANDRIĆ, F., HÉBERGER, K. (2015): Chromatographic and computational assessment of lipophilicity using sum of ranking differences and generalized pair-correlation. Journal of Chromatography A 1380(6): 130-138.

BAJUsz, D. RÁCZ, A., HÉBERGER, K. (2015): Why is Tanimoto index an appropriate choice for fingerprint-based similarity calculations? Journal of Cheminformatics 7(20): 1-13.

Boros, I. F., KAPPEL, N., GERE, A., Sipos, L. (2016): Comparison of different lettuce types (Lactuca sativa L.) based on their bioactive compounds. Proceedings of 1st International Conference on Biosystems and Food Engineering. pp. 1-8.

Csambalik L., Divéky-Ertsey, A., Pusztai, P., Boros, F., Orbán, Cs., Kovács, S., GERE, A., SiPOS, L. (2017): Multi-perspective evaluation of phytonutrients - case study on tomato landraces for fresh consumption. Journal of Functional Foods 33: 211-216.

Fragkaki, A., FARMAKi, E., ThOMAIDIS, N. S., GeOrgakopoulos, C. (2012): Comparison of multiple linear regression, partial least squares and artificial neural networks for 
prediction of gas chromatographic relative retention times of trimethylsilylated anabolic androgenic steroids. Journal of Chromatography A 1256(21): 232-239.

Gere, A., DANner, L., ANTONI, N., KovÁCS, S., DÜRrschmid, K., Sipos, L. (2016): Visual attention accompanying food decision process: an alternative approach to choose the best model. Food Quality and Preference 51: 1-7.

Gere, A., Sipos, L., KovÁCS, S., KÓKAI, Z., HÉBERger, K. (2017). Which just-about-right feature should be changed if evaluations deviate? A case study using sum of ranking differences. Chemometrics and Intelligent Laboratory Systems 161: 130-135.

HÉBERGER, K. (2010): Sum of ranking differences compares methods or models fairly. TrAC Trends in Analytical Chemistry 29(1): 101-109.

Héberger, K., KolareviĆ, S., Kračun-Kolarević, M., Sunjog, K., GaČIĆ, Z., Kluajić, Z., MitriĆ, M., VuKOviĆ-GaČIĆ, B. (2014): Evaluation of single-cell gel electrophoresis data: Combination of variance analysis with sum of ranking differences. Mutation Research-Genetic Toxicology And Environmental Mutagenesis 771: 15-22.

HÉBERGER, K., Kollár-HuneK, K. (2011): Sum of ranking differences for method discrimination and its validation: comparison of ranks with random numbers. Journal of Chemometrics 25(4): 151-158.

Kollár-HuneK, K., HÉBerger, K. (2013): Method and model comparison by sum of ranking differences in cases of repeated observations (ties). Chemometrics and Intelligent Laboratory Systems 127: 139-146.

KollÁR-HuneK, K., HÉBERGER, K. (2015): Classification of SRD-with-ties probability distributions. Conferentia Cemometrica 2015. Budapest, MTA TTK 2015. 09. 13-16. Research Centre for Natural Sciences Chemometrics and Chemoinformatics Working Group of the Hungarian Academy of Sciences.

Losó, V., Tóth, A., Gere, A., Heszberger, J., SzéKely, G., KóKAi, Z., Sipos, L. (2012): Methodology problems of the industrial preference mapping. Acta Alim. 41: 109-119.

RÁcz, A., PAPP, N., BAlogh, E.†, Fodor, M., HÉBERGer, K. (2015): Comparison of antioxidant capacity assays with chemometric methods. Analytical Methods 7(10): 42164224.

Sipos, L., Bernhardt, B., Gere, A., Komáromi, B., Orbán, Cs., Bernáth, J., Szabó, K. (2016): Multicriteria optimization to evaluate the performance of Ocimum basilicum L. varieties. Industrial Crops and Products 94: 514-519.

Sipos, L., Kovács, Z., SzÖllösi, D., KóKai, Z., Dalmadi, I., Fekete, A. (2011): Comparison of novel sensory panel performance evaluation techniques with e-nose analysis integration. Journal of Chemometrics 25(5): 275-286.

Sipos, L., Orbán, Cs., BÁlint, I., Csambalik, L., Divéky-Ertsey, A., Gere, A. (2017): Colour parameters as indicators of lycopene and antioxidant activity traits of cherry tomatoes (Solanum lycopersicum L.) European Food Research and Technology 243(5): 111.

ŠKRBić, B., HÉBERger, K., DJURiŠIĆ-MLAdENOVIĆ, N. (2013): Comparison of multianalyte proficiency test results by sum of ranking differences, principal component analysis, and hierarchical cluster analysis. Anal. Bioanal. Chem. 405: 8363-8375.

SzÖLlÖSI, D., DÉnES, D. L., FIRTHA, F., KOVÁCS, Z., FeKETE, A. (2012): Comparison of six multiclass classifiers by the use of different classification performance indicators. Journal of Chemometrics 26(3-4): 76-84.

TABACHNiCK, B. G., \& FIDELL, L. S. (2003): Preparatory data analyses. Paper presented at the annual meeting of the Western Psychological Association, Vancouver, BC, Canada. TABACHNICK, B. G., \& FIDELL, L. S. (2007): Using multivariate statistics (5th ed.) Boston, MA: Allyn \& Bacon. 